\title{
Homogenization in Stochastic Differential Geometry ${ }^{1)}$
}

\author{
By
}

\author{
Mark A. PINSKY*
}

\section{\$1. Inntroduction}

It is well known that a diffusion process on Euclidean space can be rigorously considered as the limit of a sequence of transport processes. This idea, which dates at least to Rayleigh's problem of random flight [3] has now received a general treatment by modern probabilistic methods $[2,10]$. In addition, we have shown that a suitable transport approximation remains valid for the Brownian motion of any complete Riemannian manifold [11].

In another direction several authors $[1,4,5,6,7,8]$ have considered "stochastic parallel displacement", i.e. parallel displacement of vectors along Brownian motion curves in a manifold. The purpose of this paper is to show that the stochastic parallel displacement can be rigorously considered as the limit of parallel displacement along the paths of a transport process. The transport approximation introduces an extra velocity variable which disappears in the limit, hence the term homogenization.

In addition to its elementary geometric appeal, our approach has the advantage of producing the following coordinate-free definition of the infinitesimal operator of the stochastic parallel displacement:

$$
\text { Hf }=P Z^{2} f \text {. }
$$

$\mathcal{A}$ is a second-order degenerate elliptic operator on the bundle of $k$-frames of the given manifold, $Z$ is a horizontal vector field on the bundle of

Communicated by H. Araki, Februay 8, 1980.

* Department of Mathematics, College of Arts and Sciences, Northwestern University, Evanston, 1llinois, 60201, U.S.A.

1) Work done while the author was Visiting at the University of Chicago. Supported by NSF Grant MCS 78-02144. 
$(k+1)$-frames and $P$ is the operator which averages out the extra velocity variable. In case $k=0$, our formula reduces to a multiple of the Laplace-Beltrami operator on functions [11]. In case $k=1$, our coordinate formula for $\mathcal{A}$ agrees with previously obtained formula [1] for stochastic parallel displacement. In case $k=n$ and we work with $O(M)$, the bundle of orthonormal frames, $\mathcal{A}$ is a multiple of the horizontal Laplacian [8] on $O(M)$. Formula (1.1) allows a rapid proof that this process preserves the inner product of tangent vectors.

\section{$\S 2 a$. Transport Process on the Frame Bundle}

Let $M$ be a complete Riemannian manifold, $T^{(k+1)}(M)$ the bundle of frames over $M$;

$$
T^{(k-1)}(M)=\left\{\left(x, \xi, \eta_{1}, \cdots, \eta_{k}\right): x \in M, \xi \in M_{x}, \eta_{1} \in M_{x}, \cdots, \eta_{k} \in M_{x}\right\} .
$$

Here $0 \leqq k, n$ is the dimension of $M$, and $M_{x}$ is the tangent space at $x$. Let $\gamma_{x, \xi}$ be the geodesic on $M$ with $\gamma(0)=\mathrm{x}, \dot{\gamma}(0)=\xi$. Let $\bar{\eta}_{j}(t)=\bar{\eta}\left(t ; \eta_{j}\right)$ be the parallel displacement of the tangent vector $\eta_{j}$ along $\gamma$. The canonical horizontal vector field $Z$ is defined by

$$
Z f\left(x, \xi, \eta_{1}, \cdots, \eta_{k}\right)=\left.\frac{d}{d t} f\left(\gamma(t), \dot{\gamma}(t), \bar{\eta}_{1}(t), \cdots, \bar{\eta}_{k}(t)\right)\right|_{t=0} .
$$

The projection operator $P$ is defined by

$$
\operatorname{Pf}\left(x, \eta_{1}, \cdots, \eta_{k}\right)=\int_{M_{x}} f\left(x, \xi, \eta_{1}, \cdots, \eta_{k}\right) \mu_{x}(d \xi)
$$

where $\mu_{x}(d \bar{\xi})$ is the unique rotationally invariant probability measure on the unit sphere of the tangent space $M_{x}$. Note that

$$
\begin{array}{cc}
P f=f & f \in C\left(T^{(k)}(M)\right) \\
P Z f=0 & f \in C\left(T^{(k)}(M)\right)
\end{array}
$$

where $f$ is independent of $\xi$.

Let $\left\{e_{n}\right\}_{1}^{\infty}$ be a sequence of independent random variables on a probability space $\Omega_{1}$ with the common exponential distribution

$$
\operatorname{Prob}\left\{e_{n}>t\right\}=e^{-t} \quad n=1,2, \cdots, t>0,
$$

and let $\tau_{n}=e_{1}+\cdots+e_{n}$. Define a sequence of $T^{(k+1)}(M)$-valued random 
variables $\left(x^{(n)}, \xi^{(n)}, \eta_{1}^{(n)}, \cdots, \eta_{k}^{(n)}\right) n=0,1,2, \cdots$, as follows:

$$
x^{(0)}=x, \quad \xi^{(0)}=\xi, \quad \eta_{1}^{(0)}=\eta_{1}, \cdots, \eta_{k}^{(0)}=\eta_{k} .
$$

If $\left(x^{(n)}, \xi^{(n)}, \eta_{1}^{(n)}, \cdots, \eta_{k}^{(n)}\right)$ have been defined, we let

$$
x^{(n+1)}=\gamma_{x^{(n)}, \xi^{(n)}}\left(e_{n+1}\right), \quad \eta_{j}^{(n+1)}=\bar{\eta}\left(e_{n+1} ; \eta_{j}^{(n)}\right), \quad j=1, \cdots, k .
$$

Finally $\xi^{(n}{ }^{1)}$ is distributed according to $\mu_{x^{(n+1)}}(d \xi)$, independent of $\left\{x^{(0)}\right.$, $\left.\cdots, \eta_{k}^{(n)}\right\}$. We let

$$
\begin{aligned}
& x(\iota)=\gamma_{x^{(n)}, \xi^{(n)}}\left(t-\tau_{n}\right)\left(\tau_{n} \leqq t<\tau_{n+1}\right) \\
& \xi(t)=\dot{\gamma}_{x^{(n)}, \xi^{(n)}}\left(t-\tau_{n}\right)\left(\tau_{n} \leqq t<\tau_{n+1}\right) \\
& \eta_{j}(t)=\bar{\eta}\left(t-\tau_{n} ; \eta_{j}\right)\left(1 \leqq j \leqq k, \tau_{n} \leqq t<\tau_{n+1}\right) \\
& T_{t}^{\circ} f\left(x, \xi, \eta_{1}, \cdots, \eta_{k}\right)=f\left(\gamma(t), \dot{\gamma}(t), \bar{\eta}_{1}(t), \cdots, \bar{\eta}_{k}(t)\right), \quad f \in C, \\
& R_{\lambda}^{\circ} f\left(x, \xi, \eta_{1}, \cdots, \eta_{k}\right)=\int_{0}^{\infty} e^{-\lambda t} T_{t}^{\circ} f\left(x, \xi, \eta_{1}, \cdots, \eta_{k}\right) d t, \quad f \in C, \\
& T_{t} f\left(x, \xi, \eta_{1}, \cdots, \eta_{k}\right)=E f\left(x(t), \xi(t), \eta_{1}(t), \cdots, \eta_{k}(t)\right), f \in C, \\
& R_{\lambda} f\left(x, \xi, \eta_{1}, \cdots, \eta_{k}\right)=\int_{0}^{\infty} e^{-\lambda t} T_{t} f\left(x, \xi, \eta_{1}, \cdots, \eta_{k}\right) d t, \quad f \in C,
\end{aligned}
$$

where $C$ is the space of differentiable functions on $T^{(k-1)}(M)$ which vanish at infinity.

Lemma 1. $R_{\lambda}^{0}$ maps $C$ into $C$ and $(\lambda-Z) R_{\lambda}^{0} f=\int, \int \in C$.

Proof. The geodesic flow and parallel displacement depend smoothly on initial conditions, hence the first statement. The second statement is obtained by Laplace transform.

Lemma 2. $R_{\lambda} J=R_{1+\lambda}^{0} f+R_{1+\lambda}^{0} P R_{\lambda} f, \quad f \in C$.

Proof. We use the renewal method, applied to $\tau_{1}$. Thus

$$
R_{\lambda} f=E\left\{\int_{0}^{r_{1}}+\int_{\tau_{1}}^{\infty}\right\} e^{-\lambda t} f\left(x(t), \xi(t), \eta_{1}(t), \cdots, \eta_{k}(t)\right) d t .
$$

The first term is 


$$
\begin{aligned}
E & \int_{0}^{\infty} I_{\left(t<\tau_{1}\right)} e^{-\lambda t} f\left(x(t), \xi(t), \eta_{1}(t), \cdots, \eta_{k}(t)\right) d t \\
& =\int_{0}^{\infty} e^{-t} e^{-\lambda t} f\left(\gamma(t), \dot{\gamma}(t), \bar{\eta}_{1}(t), \cdots, \bar{\eta}_{k}(t)\right) d t \\
& =R_{1+\lambda}^{0} f .
\end{aligned}
$$

The second term is

$$
\begin{aligned}
E & \int_{\tau_{1}}^{\infty} e^{-\lambda t} f\left(x(t), \xi(t), \eta_{1}(t), \cdots, \eta_{k}(t)\right) d t \\
& =E \int_{0}^{\infty} e^{-\lambda\left(\tau_{1}+s\right)} f\left(x\left(\tau_{1}+s\right), \xi\left(\tau_{1}+s\right), \cdots, \eta_{k}\left(\tau_{1}+s\right)\right) d s \\
& =E\left\{e^{-\lambda \tau_{1}} E \int_{0}^{\infty} e^{-\lambda s} f\left(x\left(\tau_{1}+s\right), \xi\left(\tau_{1}+s\right), \cdots, \eta_{k}\left(\tau_{1}+s\right)\right) d s\right\} \\
& =E\left\{e^{-\lambda \tau_{1}} E\left\{\int_{0}^{\infty} e^{-\lambda s} f\left(x\left(s ; x_{1}^{(1)}\right), \cdots, \eta_{k}\left(s ; \eta_{k}^{(1)}\right) \mid \tau_{1}, \xi_{1}\right\}\right\}\right. \\
& =E\left\{e^{-\lambda \tau_{1}}\left\{R_{\lambda} f\left(x^{(1)}, \xi^{(1)}, \eta_{1}^{(1)}, \cdots, \eta_{k}^{(1)}\right)\right\}\right\} \\
& =E\left\{e^{-\lambda \tau_{1}}\left(P R_{\lambda} f\right)\left(x^{(1)}, \xi^{(0)}, \eta_{1}^{(1)}, \cdots, \eta_{k}^{(1)}\right)\right\} \\
& =\int_{0}^{\infty} e^{-\lambda t}\left(P R_{\lambda} f\right)\left(\gamma(t), \dot{\gamma}(t), \bar{\eta}_{1}(t), \cdots, \bar{\eta}_{k}(t)\right) e^{-t} d t \\
& =R_{1+\lambda}^{0} P R_{\lambda} f .
\end{aligned}
$$

Lemma 3. $(\lambda-Z-P+I) R_{\lambda} f=f, \quad f \in C$.

Proof. Applying $(I+\lambda-Z)$ to Lemma 2, we have

$$
(I+\lambda-Z) R_{\lambda} f=f+P R_{\lambda} f
$$

which was to be proved.

Lemma 4. $T_{t} f-f=\int_{0}^{t}(Z+P-I) T_{s} f d s, \quad f \in C$.

Proof. The Laplace transform of the left-hand side is $R_{\lambda} f-\lambda^{-1} f$ while the right-hand side transforms into

$$
\int_{0}^{\infty} e^{-\lambda t} \int_{0}^{t}(Z+P-I) T_{s} f d s
$$




$$
\begin{aligned}
& =\lambda^{-1} \int_{0}^{\infty} e^{-\lambda s}(Z+P-I) T_{s} f d s \\
& =\lambda^{-1}(Z+P-I) R_{\lambda} f .
\end{aligned}
$$

Using Lemma 3, we have proved the result by uniqueness of Laplace transforms.

We now observe that we may interchange the order of the two operators appearing in the right-hand side of Lemma 4 . Indeed, from the semi-group property of $T_{t}$, it follows that $\lim _{s \rightarrow 0} s^{-1}\left\{T_{t-s} f-T_{t} f\right\}=T_{t}\left\{\lim _{s \rightarrow 0}\right.$ $\left.s^{-1}\left(T_{s} f-f\right)\right\}=T_{t}(Z+P-I) f$, from Lemma 4. Applying the fundamental theorem of calculus gives the stated result.

\section{§ 2b. Convergence to a Diffusion Process}

Let $\varepsilon>0$ be a small parameter. If we replace $Z$ by $\varepsilon Z$ in the construction of the previous section, we obtain a process

$$
\left({ }^{\varepsilon} x(t),{ }^{\varepsilon} \xi(t),{ }^{\varepsilon} \eta_{1}(t), \cdots,{ }^{\varepsilon} \eta_{k}(t)\right) .
$$

Define

$$
\begin{aligned}
& { }^{\varepsilon} T_{t} f\left(x, \xi, \eta_{1}, \cdots, \eta_{k}\right)=E\left\{f\left({ }^{\varepsilon} x\left(t / \varepsilon^{2}\right),{ }^{\varepsilon} \xi\left(t / \varepsilon^{2}\right), \cdots,{ }^{\varepsilon} \eta_{k}\left(t / \varepsilon^{2}\right)\right)\right\} \\
& { }^{\varepsilon} R_{\lambda} f\left(x, \xi, \eta_{1}, \cdots, \eta_{k}\right)=\int_{0}^{\infty} e^{-\lambda t}\left({ }^{\varepsilon} T_{t} f\right)\left(x, \xi, \eta_{1}, \cdots, \eta_{k}\right) d t
\end{aligned}
$$

It is readily verified that these correspond to the infinitesimal operator $\varepsilon^{-1} Z+\varepsilon^{-2}(P-I)$ in Lemma 4 above. We now introduce the infinitesimal operator of stochastic parallel displacement on $T^{(k)}(M)$,

$$
A=P Z^{2} \text {. }
$$

Using [5] it can be shown that $\mathcal{A}$ generates a strongly continuous semigroup of contraction operators on $C\left(T^{(k)} M\right)$. The resolvent operator is clefined by

$$
W_{\lambda} \int=\left(\lambda-P Z^{2}\right)^{-1} \int
$$

Theorem 1. If $g \in C\left(T^{(k)}(M I)\right)$, then

$$
\lim _{\varepsilon \rightarrow 0} \varepsilon R_{\lambda} g=W_{\lambda}^{r} g \text {. }
$$


We follow the analytic method of Papanicolaou [9]. For this purpose, let $f \in C^{3}\left(T^{(k)}(M)\right)$ and let

$$
f_{\varepsilon}=f+\varepsilon f_{1}+\varepsilon^{2} f_{2}
$$

where

$$
f_{1}=Z f, f_{2}=Z^{2} f-P Z^{2} f .
$$

Note that $f_{1}, f_{2} \notin C\left(T^{(k)}(M)\right)$ in general.

Lemma 5. $\left[\varepsilon^{-1} Z+\varepsilon^{-2}(P-I)\right] f_{\varepsilon}=P Z^{2} f+\varepsilon Z f_{2}, \quad \varepsilon>0$

Proof. Multiply out the six terms involved and collect like powers of $\varepsilon$. The coefficient of $\varepsilon^{-2}$ is $P f-f=0$, by (2.3). The coefficient of $\varepsilon^{-1}$ is $Z f+(P-I) f_{1}=Z f+(P-I) Z f=Z f-Z f=0$, by (2.4). The constant term is $Z f_{1}+(P-I) f_{2}=Z^{2} f+(P-I)\left(Z^{2} f-P Z^{2} f\right)=Z^{2} f+P Z^{2} f-P Z^{2} f$ $-Z^{2} f+P Z^{2} f=P Z^{2} f$. Finally the coefficient of $\varepsilon$ is just $Z f_{2}$.

Proof of the Theorem. We write Lemma 4, rescaled with $\varepsilon$, in terms of Laplace transforms. Thus

$$
{ }^{\varepsilon} R_{\lambda} f_{\varepsilon}-\lambda^{-1} f_{\varepsilon}={ }^{\varepsilon} R_{\lambda}\left(\varepsilon^{-1} Z+\varepsilon^{-2}(P-I)\right) f_{\varepsilon} .
$$

Using Lemma 5 and collecting terms, we have

$$
{ }^{\varepsilon} R_{\lambda}\left(\lambda f-P Z^{2} f\right)=f+\varepsilon F_{1}+\varepsilon^{2} F_{2}
$$

where

$$
\begin{aligned}
& F_{1}=f_{1}-{ }^{\varepsilon} R_{\lambda} f_{1}-{ }^{\varepsilon} R_{\lambda} Z f_{2} \\
& F_{2}=f_{2}-\lambda^{\varepsilon} R_{\lambda} f_{2} .
\end{aligned}
$$

Now let $\lambda f-P Z^{2} f=g, f=W_{k} g$. Letting $\varepsilon \rightarrow 0$, we have proved that $\lim _{\varepsilon \rightarrow 0}{ }^{\varepsilon} R_{\lambda} g=W_{\lambda} g$, as required.

We now justify the term "stochastic parallel displacement". Let $X(t)$ be the diffusion process on $T^{(k)}(M)$ governed by the differential operator $P Z^{2}$. Let $N_{i j}=\left(\eta_{i}, \eta_{j}\right)$ be the inner product of a pair of tangent vectors, $1 \leqq i, j \leqq k . \quad N_{i j}$ is a real valued function on $T^{(k)}(M)$.

Theorem 2. $N_{i j}(X(t))=N_{i j}(X(0)), 1 \leqq i, j \leqq k, t \geqq 0$. 
For the proof we first note the useful

Lemma 6. $Z N_{i j}=0$.

Proof. The classical parallel displacement preserves the inner product of tangent vectors. Thus $\left(\bar{\eta}_{i}(t), \bar{\eta}_{j}(t)\right)=\left(\bar{\eta}_{i}(0), \bar{\eta}_{j}(0)\right)$. Glancing at (2.1) shows that $Z N_{i j}=0$.

Lemma 7. $N_{i j}(X(t))$ is a martingale.

Proof. It suffices to show that $P Z^{2}\left(N_{i j}\right)=0$, which is immediate from Lemma 6.

Lemma 8. The increasing process of $N_{i j}(X(\iota))$ is zero.

Proof. By the results of Taylor [12] for example, it suffices to show that $P Z^{2}\left(N_{i j}^{2}\right)-2 N_{i j} P Z^{2}\left(N_{i j}\right)=0$. But this also follows immediately from Lemma 6.

\section{§3. Explicil Formulas in Local Coordinates}

The operator $P Z^{2}$ which occurs in the above limit theorem is called the homogenized transport operator. It is an invariantly defined second order differential operator on the frame bundle $T^{(k)}(M)$. In case $k=0$, we have shown [11] that the homogenized transport operator is equal to $n^{-1}$ times the Laplace-Beltrami operator of the Riemannian metric. We now obtain an explicit local formula in case $k=1$, i.e. the tangent bundle. For this purpose, we work with the bundle of 2-frames

$$
T^{(2)}(M)=\left\{(x, \tilde{\xi}, \eta): x \in M, \tilde{\xi} \in M_{x}, \eta \in M_{x}\right\}
$$

Let $\gamma(t)$ be the geodesic with $\gamma(0)=x, \dot{\gamma}(0)=\xi$. Let $\eta(t)$ be the parallel displacement of $\eta$ along $\gamma$. The canonical horizontal vector field $Z$ is defined by

$$
Z f(x, \xi, \eta)=\left.\frac{d}{d t} f(x(t), \xi(t), \eta(t))\right|_{t=0}
$$


In a coordinate chart we can compute $Z$ by the formula

$$
Z_{f}(x, \xi, \eta)=\xi^{i} \frac{\partial f}{\partial x}-\Gamma_{i j}^{k} \xi^{i} \xi^{j} \frac{\partial f}{\partial \xi^{k}}-\Gamma_{i j}^{k} \xi^{i} \eta^{j} \frac{\partial f}{\partial \eta^{k}} .
$$

The projection operator $P$ maps from $C\left(T^{(2)}(M)\right)$ to $C(T(M))$ by the rule

$$
P f(x, \xi)=\int_{M_{x}} f(x, \xi, \eta) \mu_{x}(d \xi)
$$

where $\mu_{x}$ is the rotationally invariant probability measure on the unit sphere of $M_{x}$. Of particular relevance are the coordinate formulas [11].

$$
P\left(\xi^{i} \xi^{j}\right)=n^{-1} g^{i j}
$$

where $g^{i j}$ is the inverse of the metric tensor. The homogenized transport operator can be computed according to the following

Proposition 9. In a coordinate chart we have the formula

$$
\begin{gathered}
P Z^{2} f=n^{-1} g^{i l}\left\{f_{x^{i} x^{l}}+\Gamma_{i j}^{k} \Gamma_{l m}^{n} \eta^{j} \eta^{m} f_{\eta^{k} \eta^{n}}-2 \Gamma_{l m}^{n} \eta^{m} f_{x^{i} \eta^{n}}\right. \\
\left.-\Gamma_{i l}^{j} f_{x^{j}}+\left(\Gamma_{i l}^{j} \Gamma_{j m}^{n} \eta^{m}+\Gamma_{i j}^{k} \Gamma_{l k}^{n} \eta^{j}-\Gamma_{l m, i}^{n} \eta^{m}\right) f_{\eta^{n}}\right\}
\end{gathered}
$$

where $f=f(x, \eta)$ is a $C^{2}$ function.

Proof. This is a straightforward computation, using (3.1), (3.2), (3. 4). Except for the sign convention of $\Gamma_{i j}^{k}$, this agrees with the formulas of $[1,4]$.

We now consider the case $k=n$, specializing to $O(M)$ the bundle of orthonormal frames. Let $\left(e_{1}, \cdots, e_{n}\right)$ be an orthonormal basis of $M_{x}$. Let $\gamma_{\beta}(t)$ be the geodesic with $\gamma_{\beta}(0)=x, \dot{\gamma}_{\beta}(0)=e_{\beta}$. Let $e_{i \beta}(t)$ be the parallel displacement of $e_{i}$ along $\gamma_{\beta}$. The horizontal vector field $E_{\beta}$, $1 \leqq \beta \leqq n$, is defined by

$$
E_{\beta} f=\left.\frac{d}{d t} f\left(\gamma_{\beta}(t), e_{1 \beta}(t), \cdots, e_{n \beta}(t)\right)\right|_{t=0} .
$$

The horizontal Laplacian is defined by

$$
\Delta_{O(M)} f=\sum_{\beta=1}^{n} E_{\beta}^{2} f
$$


Proposition 10. $\Delta_{o(M)} f=\left.n P Z^{2} f\right|_{o(M)}, f \in C\left(T^{k}(M)\right)$.

Proof. In any coordinate chart, we have

$$
Z f=\xi^{i} \frac{\partial f}{\partial x^{i}}-\Gamma_{i j}^{k} \xi^{i} \xi^{j} \frac{\partial f}{\partial \xi^{k}}-\Gamma_{i j}^{k} \xi^{i} \eta_{\alpha}^{j} \frac{\partial f}{\partial \eta_{\alpha}^{k}} .
$$

Assuming a normal chart centered at $x$, we have for $f \in C\left(T^{(k)}(M)\right)$

$$
\begin{aligned}
Z^{2} f & =\xi^{l} \frac{\partial}{\partial x^{l}}\left\{\xi^{i} \frac{\partial f}{\partial x^{i}}-\Gamma_{i j}^{k} \xi^{i} \eta_{\alpha}^{j} \frac{\partial f}{\partial \eta_{\alpha}^{k}}\right\} \\
& =\xi^{i} \xi^{l} \frac{\partial^{2} f}{\partial x^{i} \partial x^{l}}-\Gamma_{i j, l}^{k} \xi^{l} \xi^{i} \eta_{\alpha}^{j} \frac{\partial f}{\partial \eta_{\alpha}^{k}}
\end{aligned}
$$

Using (3.4)

$$
P Z^{2} f=n^{-1}\left[\frac{\partial^{2} f}{\partial x^{i} \partial x^{i}}-\Gamma_{i j, i}^{k} \eta_{\alpha}^{j} \frac{\partial f}{\partial \eta_{\alpha}^{k}}\right]
$$

To compare with (3.7), we recall the coordinate form of (3.6) [5]:

$$
E_{\beta} f=e_{\beta}^{i} \frac{\partial f}{\partial x^{i}}-e_{\beta}^{i} e_{\alpha}^{j} \Gamma_{i j}^{k} \frac{\partial f}{\partial\left(e_{\alpha}^{k}\right)}
$$

where $\left(x^{1}, \cdots, x^{n}, e_{1}^{1}, e_{1}^{2}, \cdots, e_{n}^{n}\right)$ is any coordinate chart on $O(M)$. Assuming a normal chart centered at $x$, we have

$$
\begin{aligned}
E_{\beta}^{2} f & =e_{\beta}^{i} \frac{\partial}{\partial x^{i}}\left\{e_{\beta}^{i} \frac{\partial f}{\partial x^{i}}-e_{\beta}^{i} e_{\alpha}^{j} \Gamma_{i j}^{k} \frac{\partial f}{\partial\left(e_{\alpha}^{k}\right)}\right\} \\
& =e_{\beta}^{l} e_{\beta}^{i} \frac{\partial^{2} f}{\partial x^{i} \partial x^{i}}-e_{\beta}^{i} e_{\beta}^{l} e_{\alpha}^{j} \Gamma_{i j, l}^{k} \frac{\partial f}{\partial\left(e_{\alpha}^{k}\right)}
\end{aligned}
$$

Summing on $\beta$ and using the orthonormality, we have

$$
\sum_{\beta=1}^{n} E_{\beta}^{2} f=\frac{\partial^{2} f}{\partial x^{i} \partial x^{i}}-e_{\alpha}^{j} \Gamma_{i j, i}^{k} \frac{\partial f}{\partial\left(e_{\alpha}^{k}\right)}
$$

which agrees with (3.9) to within the factor $n^{-1}$.

Finally, we note that the orthonormal frame $m=\left(x, e_{1}, \cdots, e_{n}\right)$ has a unique stochastic parallel displacement along the Brownian motion path. For this purpose, recall the Stratanovich equation [5] for horizontal diffusion on $O(M)$ :

$$
d m=\sum_{\beta=1}^{n} E_{\beta} \cdot d w^{\beta}
$$


Using the coordinate representation (3.10), we have

$$
\begin{aligned}
d x^{i} & =e_{\beta}^{i} \cdot d w^{\beta} \\
d e_{\alpha}^{k} & =-e_{\beta}^{i} e_{\alpha}^{j} \Gamma_{i j}^{k} \cdot d w^{\beta} \\
& =-e_{\alpha}^{i} \Gamma_{i j}^{k} \cdot d x^{i} .
\end{aligned}
$$

Thus, given the Brownian motion path $\{x(t), t \geqq 0\}$, the vector $e_{\alpha}(t)$ is uniquely determined by solving a linear system of stochastic differential equations. Thus we have proved

Proposition 11. The mapping $\left(x(t), e_{1}(t), \cdots, e_{n}(t)\right) \rightarrow x(t)$ is a measure-preserving bijection from the path space of the horizontal diffusion on $O(M)$ to the path space of the Brownian motion on $M$.

\section{References}

[1] Airault, H., Subordination de processus dans le fibre tangent et formes harmoniques, Comptes Rendus Academie des Sciences, Série A, 282 (1976), 1311-1314.

[2] Bensoussan, A., Lions, J. and Papanicolaou, G., Boundary layers and homogenization of transport processes, Publ. RIMS, Kyoto Univ., 15 (1979), 53-158.

[3] Chandresekhar, S., Stochastic Problems in Physics and Astronomy, Rev. Modern Physics, 15 (1943).

[4] Dynkin, E. B., Diffusion of Tensors, Dokl. Akad. Nauk, SSSR, 179 (1968), 532-535.

[5] Ikeda, N. and Watanabe, S., Stochastic Differential Equations, Kodansha, 1981.

[6] Itô, K., The Brownian motion and tensor fields on Riemannian manifolds, International Congress of Mathematicians, Stockholm, (1962), 536-539.

[7] - Stochastic parallel displacement, Lecture Notes in Math., Springer Verlag, 451, 1-7.

[8] Malliavin, P., Formules de la moyenne, pour les formes harmoniques, J. Fun. Anal., 17 (1974), 274-291.

[9] Papanicolaou, G., Probabilistic Problems and Methods in Singular Perturbations, Rocky Mountain J. Math., 6 (1976), 653-673.

[10] Papanicolaou, G., Stroock, D. and Varadhan, S., A martingale approach to some limit theorems, Duke Univ. Turbulence Conference, 1977.

[11] Pinsky, M., Isotropic transport process on a Riemannian manifold, Trans. Amer. Math. Soc., 218 (1976), 353-360.

[12] Taylor, J. C., The comparison lemma of Malliavin and related topics, Seminaire de Prob., Strasbourg, 1977-78, 446-455. 